and combination tones depending upon the type of perturbation. As this is unknown, all that can be said is that the maximum effect will be of the order of the nonlinear terms in the development of the polarizability. The effect will therefore be small in general.

In conclusion, it may be said that the appearance of Raman lines is governed by the following factors: symmetry of the molecule, the effect of the motion of the hydrogens relative to carbon on the magnitude of $\alpha_{j}$, and $\alpha_{i j}$, resonance interaction of nearly commensurable frequencies, anharmonicity of the vibrations involved. In the case of the isotopic methanes, the nonappearance of fundamentals may be attributed to the circumstance that $\alpha$, is very small. The appearance of overtones may be due either to the fact that $\alpha_{j j}$ is large, or to resonance, or their combined influence. The effect of anharmonicity should be small, but cannot be predicted.

\title{
An Analytic Method of Interpretation of Electron Diffraction Photographs of Gases*
}

\author{
S. H. BAUER, California Institute of Technology
}

(Received March 24, 1936)

An analytic procedure to be used in the interpretation of electron diffraction photographs for gases is developed. The experimentally determined positions of the maxima and minima are employed to solve directly for the interatomic distances through successive approximations. The method is then generalized so as to be applicable to molecules in which rotations or large oscillations about a bond are

\section{I}

$I^{2}$ $\mathrm{N}$ the analysis of electron or $\mathrm{x}$-ray diffraction photographs of gases, it has been customary to plot $I$ versus $x$, of the function

$$
I=k \sum_{i j} \psi_{i} \psi_{j} \frac{\sin l_{\imath_{j}} x}{l_{i j} x},
$$

where

$$
\begin{aligned}
\psi_{\imath \text { x-rays }} & =f_{i}(x) \text { the structure factor for atom } i \\
\psi_{i \text { elcetrons }} & =4 \pi\left(Z_{\imath}-f_{\imath}(x)\right) / x^{2} \\
x & =4 \pi \sin (\theta / 2) / \lambda . \\
l_{i j} & =\text { distance between the } i \text { th and } j \text { th } \\
& \text { atoms, }
\end{aligned}
$$

for various values of the parameters $l_{23}$, and to choose those values for which the corresponding curve shows the best fit with the experimentally determined intensity distribution both in general shape and in the positions of the maxima and

* Contributions from Gates Chemical Laboratory, California Institute of Technology, No. 540. permitted. As a test, three sets of data which have already been published are recalculated yielding somewhat altered values for the molecular parameters. New diffraction photographs of propane are analyzed. It is pointed out that the number of theoretical intensity curves which must be computed to obtain the correct structure is thereby greatly reduced.

minima. Those investigators ${ }^{1}$ working with electron beams found it advantageous to use various simpler functions for the form of $\psi_{i}$ than the one given above. ${ }^{2}$ Justification for these has been adequately discussed in the literature. It is evident that even with the simplest form of $\psi_{2}\left(\approx Z_{2}\right)$, the labor involved in calculating the numerous curves for molecules having only three doubtful shape parameters becomes very great. Were it desirable to investigate three different values for each $l_{i j}$ in such a case, it would be necessary to plot $3^{3}$ curves for each conceivable model to take care of all possible combinations. Then, to obtain the best fit, it might be essential to interpolate between the curves. For more complicated molecules the necessary number of computations rises very rapidly. In practice, therefore, one must make frequent use of the assumption of the constancy of interatomic distances and angles for the same group of atoms in different molecules and can investigate only "reasonable" models.

${ }^{1}$ L. Pauling and L. O. Brockway, J. Chem. Phys. 2 867 (1934); L. R. Maxwell, S. B. Hendricks and Y. M. Mosley, ibid. 3, 699 (1935).

${ }^{2}$ N. F. Mott, Proc. Roy. Soc. A127, 658 (1930). 
One may regard the determination of molecular structure from electron diffraction photographs as consisting of two parts; the first, in which the form of the model is determined, the second, in which exact values for the interatomic distances are obtained. As has been indicated, in the procedures used heretofore, both parts necessitated the plotting of many intensity curves, their shape being the deciding factor in the first and the exact positions of the maxima and minima in the second. The radial distribution method $^{11}$ is a step towards the reduction of the somewhat personal factor in the analysis leading to the correct model; the following analytic procedure in which the experimentally determined $x_{\max }$ and $x_{\min }$ values are directly employed eliminates the stochastic approach to the evaluation of the interatomic distances. This method is particularly applicable to situations wherein a large number of sharp maxima and minima appear.

For the purposes of the discussion assume that the visually estimated ring diameters correspond to extremal values of the function ${ }^{3}$

$$
\sum_{\imath \jmath} Z_{i} Z_{\bar{l}} \frac{\sin l_{\imath \imath} x}{l_{\imath \jmath} x} .
$$

Their positions $\left(x_{\kappa}\right)$ are given analytically by the equation

$$
\sum_{i j} Z_{\imath} Z_{\jmath}\left(\cos l_{\imath j} x_{\kappa}-\frac{\sin l_{\imath \imath} x_{\kappa}}{l_{\imath \jmath} x_{\kappa}}\right)=0 .
$$

If there are at least as many experimental values of $x_{k}$ as there are unknown independent $l$, 's, it is possible to determine the latter by solving the set of $\kappa$ simultaneous equations given by (3). Provided the type of model chosen is the correct one, the values so obtained should be reasonable and should be in agreement with the $x_{\kappa}$ 's not used in the calculation. Unfortunately, the exact solution of the above set does not appear feasible. ${ }^{4}$

\footnotetext{
${ }^{3}$ Generalization to the case $\psi_{3} \neq Z_{2}$ will be obvious. It will then be necessary to determine $f_{2}(x)$ in analytic form. See L. Pauling and J. Sherman, Zeits. f. Krist. 81, 1 (1932). For more accurate work, this will probably become essential.

${ }_{4}$ The expansion of $(3)$ into a series

$$
\sum_{n=1}^{\infty} \frac{(-1)^{n} n x_{\kappa}^{2 n}}{(2 n+1) !}\left(\sum_{i j} Z_{i} Z_{3} l_{2 j}{ }^{2 n}\right)
$$

is of no aid whatever. Since the values of $l_{2}$, are often as large as 5 and those of $x_{\kappa}$ as high as 20 , the convergence is extremely slow.
}

This will be even more evident when it is recalled that the number of $l_{i j}$ 's occurring in (3) is, except for the simplest structures, greater than the number of independent parameters, if the obvious symmetry of the molecule is considered. Some of these will, therefore, be expressed as functions of the fewer remaining ones.

Results can be deduced through successive approximations if use is made of a table of empirical covalent radii. ${ }^{5}$ Choose reasonable values for the independent $l_{i j}$ 's $\left(l_{1,2}, \mathbf{3} \ldots n\right)$. Substitute these in (3) along with the experimentally determined $x_{\kappa}$ 's. If the former are correct, all of the $\kappa$ equations will reduce to zero; if not, each will equal some value $Y_{x}$ (referred to as residual) whose absolute magnitude is directly proportional to the departure from the correct model. In order to find the corrections $\Delta l_{1 \ldots n}$ which must be applied to the chosen $l_{1 \ldots n}$ to make them satisfy (3), differentiate the latter to obtain

$$
\begin{aligned}
& \Delta Y_{\kappa} \approx Y_{\kappa}^{\prime}-Y_{\kappa} \approx \sum_{n} \frac{\partial Y}{\partial l_{n}} \Delta l_{n}= \\
& -\sum_{n \imath j} Z_{\imath} Z_{3}\left[x_{\kappa} \sin l_{\imath j} x_{\kappa}+\frac{\cos l_{\imath j} x_{\kappa}}{l_{\imath j}}\right. \\
& \left.-\frac{\sin l_{i j} x_{\kappa}}{x_{\kappa} l_{i j}^{2}}\right]\left(\frac{\partial l_{\imath 2}}{\partial l_{n}}\right) \Delta l_{n},
\end{aligned}
$$

where the new values $Y_{x}^{\prime}$ are to vanish. Since the $Y_{x}$ 's and the differential coefficients can be readily evaluated, ${ }^{6}$ the problem reduces to the solution of a set of linear equations in $\Delta l_{n}$. For higher approximations, the new values of $l_{n}$ can be used and the process repeated. When more rings are available than there are $l_{n}$ 's, a least-squares treatment of the simultaneous group given by (4) is the logical thing to apply.

One obvious limitation of this procedure can be immediately indicated. Since the functions occurring in (3) and (4) are multivalued, the original guess must not be too distant from the correct values. Another is involved in the initial assumption since it is not yet entirely clear what part of the peak or trough is measured in the

${ }^{5}$ L. Pauling and M. L. Huggins, Zeits. f. Krist. 87, 205 (1934).

${ }^{6} \mathrm{~A}$ table or large scale plot of the functions $(\cos x-\sin$ $x / x)$ and $(x \sin x+\cos x-\sin x / x)$ greatly facilitates the calculations. 
visual method. It is certain that for peaks which have shoulders or are separated by shallow minima, measurements so obtained do not give quite the correct $x_{\max }$ 's. Allowance can be made for this effect when the appearance of the picture as well as the experimental ring diameters are compared with a complete plot of $I$ versus $x$, but it would be highly arbitrary, if indeed it were possible, to introduce a similar allowance in the analytic expressions. One can conclude, therefore, that wherever the interatomic distances are known approximately, the $x$ values of sharp maxima and minima of electron diffraction photographs may be used to obtain them precisely.

Up to the present, the treatment of molecules in which rotations or large oscillations about a bond are permitted has not been very satisfactory. ${ }^{7}$ The stochastic procedure which has been applied does not give clear-cut results; the radial distribution method ${ }^{11}$ is more reliable. The treatment outlined above can be readily extended to take care of such a situation. Since the time spent by a diffracted electron in the vicinity of the diffracting molecule is small compared to the period of a rotation, the resultant intensity distribution will be similar to one obtained from a mixture of different molecules, each species being present in a concentration proportional to the relative probability of any one specific position in the allowed cycle. Were one to write for the contribution of one species

$$
I_{s}=k^{\prime} P_{s} \sum_{i j} Z_{\imath} Z_{j} \frac{\sin l_{i j} x}{l_{i j} x}
$$

where $P_{s}$ is the probability that the interatomic distances be $l_{\imath i}$, the total intensity can be expressed by

$$
I=k^{\prime} \sum_{s i j} P_{s} Z_{i} Z_{j} \frac{\sin \alpha_{s} l_{i j} x}{\alpha_{s} l_{i j} x},
$$

where $\alpha_{s}$ are the factors by which $l_{i j}$ must be multiplied in going from one standard model to

\footnotetext{
${ }^{7}$ Symmetrical di-halogen substitution products of ethane have been discussed by: L. Meyer, Zeits. f. physik. Chemie B8, 32 (1930)-dielectric constant measurement; R. Wierl, Physik. Zeits. 31, 366 (1930), Ann. d. Physik 13, 453 (1932);-electron diffraction; F. Ehrhardt, Physik. Zeits. 33, 610 (1932)-x-ray diffraction. The only possibilities which have been considered by the latter two investigators are: (a) completely free rotation, or (b) the presence of only two species-cis and trans forms-in various concentrations.
}

any of the others. Consider at first the simple case wherein the interatomic distances of the standard one are known (i.e., $l_{i j}, \alpha_{s}$ ), it being desired to find the relative probability of a number of discrete orientations. Since the observed extremal values occur at

$\sum_{s i 2} P_{s} Z_{i} Z_{i}\left(\cos \left(\alpha_{s} l_{i j}\right) x_{\kappa}-\frac{\sin \left(\alpha_{s} l_{i j}\right) x_{\kappa}}{\left(\alpha_{s} l_{i j}\right) x_{\kappa}}\right)=0$

with the restrictions $s \leqslant k ; P_{s} \geq 0 ; \sum_{s} P_{s}=1$, it is only necessary to solve this set of linear simultaneous equations for the $P_{s}$ 's.

A more general procedure may be outlined. Actually both $P_{s}$ and $l_{i j}$ of (5) vary continuously. Let us write for

$$
\begin{aligned}
& l_{i j}=f_{2 j}\left(l_{n}, \theta_{i j}\right), \\
& d n=N P_{1}\left(l_{i j}\right) d l_{\imath j}=N P_{11}\left(\theta_{i j}\right) d \theta_{i j}
\end{aligned}
$$

with

$$
\int_{l_{\imath j^{\prime}}}^{l_{\imath j^{\prime \prime}}} P_{1}\left(l_{\imath \jmath}\right) d l_{i j}=1 \text {, }
$$

where $l_{n}$ represents the independent parameters of a standard configuration;

$\theta_{i j}$ the angle of rotation from the model chosen;

$P_{1}\left(l_{n}\right)$ the probability that $d n$ molecules have their interatomic distances between $l_{i j}$ and $l_{i j}$ $\left.+d l_{n j}\right)$

$l_{\imath \eta^{\prime}}$ and $l_{i i^{\prime}}{ }^{\prime \prime}$ are the possible extreme values of $l_{\imath y}$.

The observed intensity distribution will then be

$$
I=N k^{\prime \prime} \sum_{i j} Z_{i} Z_{j} \int_{l_{i j^{\prime}}}^{l_{i j^{\prime \prime}}} P_{1}\left(l_{i j}\right) \frac{\sin l_{i j} x}{l_{i j} x} d l_{i j}
$$

and the condition for a maximum or minimum

$$
\sum_{i j} Z_{\imath} Z_{j} \int_{l_{i y^{\prime}}}^{l_{i_{3} \prime \prime} \prime} P_{1}\left(l_{\imath \jmath}\right)\left(\cos l_{i j} x_{\kappa}-\frac{\sin l_{\imath 2} x_{\kappa}}{l_{\imath \jmath} x_{\kappa}}\right) d l_{i j}=0
$$

The above expression has meaning only when the integral can be evaluated. A general form for $P_{1}\left(l_{2_{2}}\right)$ which makes this possible is an expansion into a polynomial in $l_{i j}$ or $\left(1 / l_{i j}\right) .{ }^{8}$ Thus, if the

${ }^{8}$ See Pierce's Short Table of Integrals (1929), pp. 46-47; Jahnke-Emde, Table of Functions (1933), p. 83 for a table of $\int_{0}^{x}(\sin t / t) d t$ for $0 \leq x \leq \infty$. One is not, however, limited to the use of a power series. Functions which may be expressed as a Fourier integral involving undetermined parameters may be equally satisfactory. Since the limits of integration are thus shifted to $\pm \infty$ (the $l_{i j}^{\prime}$ and $l_{i j}{ }^{\prime \prime}$ will now appear explicitly in the functional argument of the integral) the necessary integrations with regard to $l_{i j}$ can be readily performed. 
limiting values $l_{i j}{ }^{\prime}$ and $l_{i j}{ }^{\prime \prime}$ are known, as many coefficients of the polynomial can be calculated through the solution of the simultaneous set $(7 \mathrm{~b})$, as there are experimental $x_{x}$ 's. Otherwise, the latter equation may be differentiated with respect to the limits of integration, giving

$$
\begin{aligned}
& \Delta Y_{\mathrm{k}}=\sum_{i j l_{2}{ }^{\prime \prime}} Z_{2} Z_{j} P_{1}\left(l_{2}{ }^{\prime \prime}\right)\left(\cos l_{2}{ }^{\prime \prime} x_{\mathrm{k}}-\frac{\sin l_{21}{ }^{\prime \prime} x_{\mathrm{k}}}{l_{2 \jmath}{ }^{\prime \prime} x_{\mathrm{k}}}\right) \Delta l_{2,}{ }^{\prime \prime}
\end{aligned}
$$

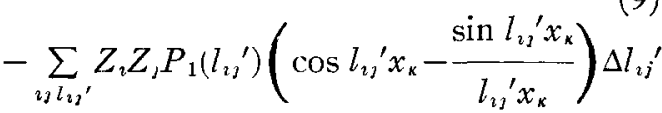

and the method of successive approximations applied. Then as many coefficients as there are $x_{x}$ 's less the number of independent unknown $l_{\imath j}^{\prime \prime}, "$ 's may be evaluated. It should be kept in mind that a different $P_{1}\left(l_{23}\right)$ function will be needed for every independently varying distance. Since the form of $f_{i j}\left(l_{n}, \theta_{\imath}\right)$ for any type of model will be known,

$$
P_{11}\left(\theta_{\imath \jmath}\right)=P_{1}\left(l_{i j}\right) \partial l_{i j} / \partial \theta_{i j}
$$

and, for the particular rotation considered, the potential energy of the molecule at any angle

$$
U\left(\theta_{\imath \jmath}\right)=-k T \log P_{11}\left(\theta_{\imath \jmath}\right)
$$

can be deduced.

\section{II}

To test the applicability of Eqs. (3) and (4), three sets of data which already have appeared in the literature have been recalculated.

\section{Methylene chloride ${ }^{9}$}

The two independent parameters taken were the carbon-chlorine and the chlorine-chlorine distances. The effects of the hydrogen atoms can be assumed to be negligible. Ten $x_{k}$ values were treated by least squares which gave for the final result

$$
\left.\begin{array}{rr}
\mathrm{C}-\mathrm{Cl} & 1.78_{5} \mathrm{~A} \\
\mathrm{Cl}-\mathrm{Cl} & 2.91_{3} \mathrm{~A} \\
(\mathrm{Cl}-\mathrm{C}-\mathrm{Cl}) & 109^{\circ}{ }_{22^{\prime}}
\end{array}\right\} \text { as contrasted with }\left\{\begin{array}{l}
1.77 \pm 0.03 \mathrm{~A} \\
2.92 \pm 0.02 \mathrm{~A} \\
111^{\circ} \pm 2^{\circ}
\end{array}\right.
$$

published by the original investigators. The accuracy claimed for the new values cannot be greater than that stated for the old, since the largest

${ }^{9}$ L. E. Sutton and L. O. Brockway, J. Am. Chem. Soc. 57,473 (1935). source of error is probably inherent in the voltage measurement. That the newly calculated distances will be in better agreement with the given data so far as positions of the ten $x_{\kappa}$ values are concerned is, however, emphasized.

\section{Chloroform ${ }^{8}$}

The hydrogen terms were again neglected. Thirteen rings were used giving for the final results:

$$
\left.\begin{array}{rr}
\mathrm{C}-\mathrm{Cl} & 1.78_{1} \mathrm{~A} \\
\mathrm{Cl}-\mathrm{Cl} & 2.91_{0} \mathrm{~A} \\
(\mathrm{Cl}-\mathrm{C}-\mathrm{Cl}) & 109^{\circ}{ }^{\circ}
\end{array}\right\} \begin{aligned}
& \text { which are to be com- } \\
& \text { pared with those of } \\
& \begin{array}{l}
\text { Sutton and Brock- } \\
\text { way, }
\end{array}
\end{aligned}\left\{\begin{array}{l}
1.78 \pm 0.03 \mathrm{~A} \\
2.93 \pm 0.02 \mathrm{~A} \\
111^{\circ} \pm 2^{\circ}
\end{array}\right.
$$

\section{Phosgene ${ }^{10}$}

The three parameters chosen were the $\mathrm{C}-\mathrm{Cl}$, $\mathrm{Cl}-\mathrm{Cl}$ and $\mathrm{C}-\mathrm{O}$ distances, it being assumed that the molecule is planar. The sums of the covalent radii were taken as the zeroth approximation and eleven rings used in a least-squares treatment of the simultaneous set of Eqs. (4). (The first two rings as well as the ones at $x_{\kappa}=12.54$ and 13.51 were discarded for it is obvious that the St. John effect would greatly disturb accurate measurement.) The resulting values gave curve $A_{1}$ of Fig. 1, which is to be compared with $B$, the final curve chosen by the above authors. The lack of an indication of a shoulder at $x_{\kappa}=13.51$ eliminates this model. Hence a second approximation (corresponding curve is $A_{2}$ ) was calculated giving for

$$
\left.\begin{array}{rl}
\mathrm{C}-\mathrm{Cl} & 1.68 \mathrm{~A} \\
\mathrm{Cl}-\mathrm{Cl} & 2.86 \mathrm{~A} \\
\mathrm{C}-\mathrm{O} & 1.21 \mathrm{~A} \\
\mathrm{Cl}-\mathrm{C}-\mathrm{Cl} & 116^{\circ}
\end{array}\right\} \text { which are to be compared with }\left\{\begin{array}{l}
1.68 \mathrm{~A} \\
2.87 \mathrm{~A} \\
1.28 \mathrm{~A} \\
117^{\circ}
\end{array}\right.
$$

$A_{2}$ is not completely satisfactory yet since the peak at $x_{\kappa}=9.34$ has almost disappeared while the one at 13.51 became unduly prominent. The similarity between the two sets of distances clearly indicates, however, the advantages of the straightforward analytic procedure, for it should be recalled that in order to arrive at the values given by Brockway, Beach and Pauling, approximately twenty-five intensity curves had to be plotted. The best agreement with the data (curve $B_{1}$ ) was obtained through another successive

${ }^{10}$ L. O. Brockway, J. Y. Beach, and L. Pauling, J. Am. Chem. Soc. 57, 2693 (1935). 


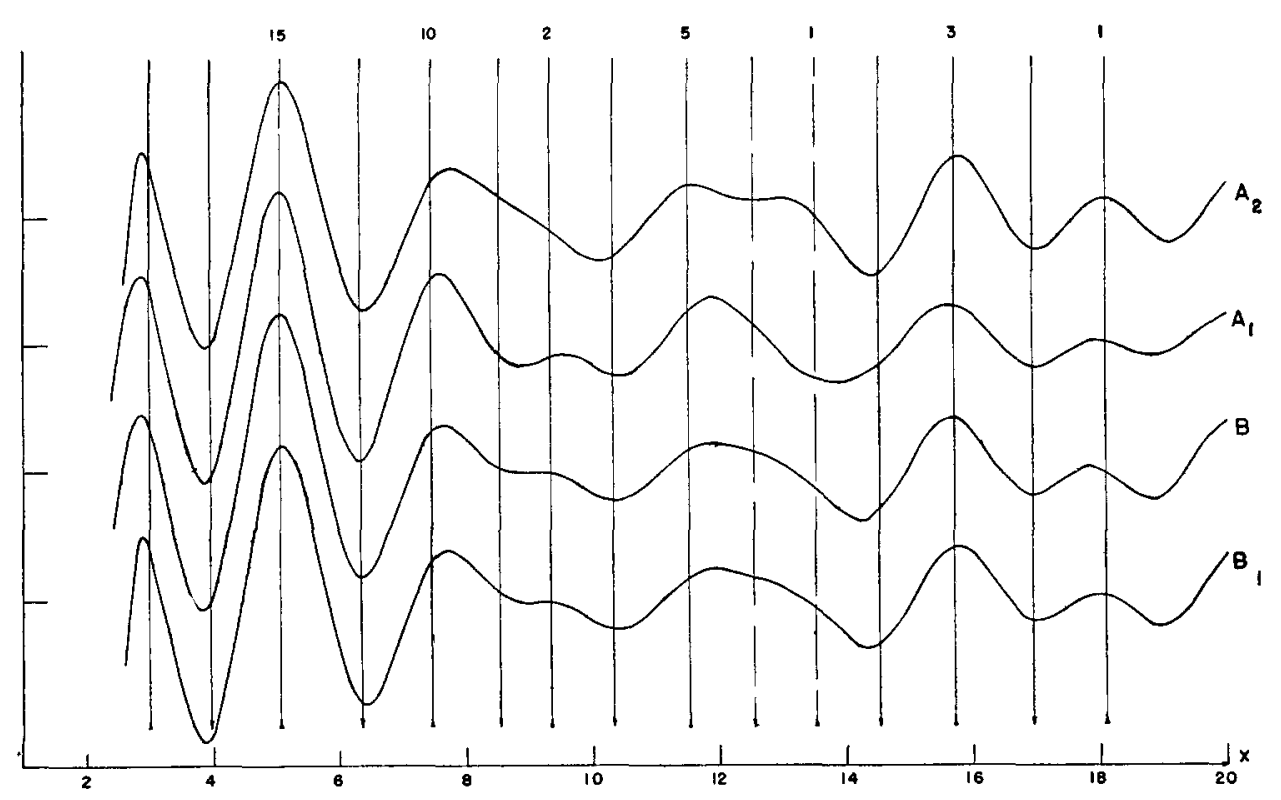

FIG. 1. Calculated intensity curves for phosgene. $A_{1}$, first approximation using the covalent radii and $114^{\circ}$ for the $\mathrm{Cl}-\mathrm{C}-\mathrm{Cl}$ angle as the zeroth approximation. $A_{2}$, second approximation to the above. $B$, final values of Brockway, Beach and Pauling. $B_{1}$, first approximation to $B$. The numbers at the top of the figure indicate the visually estimated relative intensities of the maxima.

approximation using the distances of $B$ in Eqs. (3) and (4). This resulted in

\section{Propane}

$$
\begin{array}{rr}
\mathrm{C}-\mathrm{Cl} & 1.67_{4} \mathrm{~A} \\
\mathrm{Cl}-\mathrm{Cl} & 2.85_{3} \mathrm{~A} \\
\mathrm{C}-\mathrm{O} & 1.26_{0} \mathrm{~A} \\
\mathrm{Cl}-\mathrm{C}-\mathrm{Cl} & 116^{\circ}{ }_{54}
\end{array}
$$

Electron diffraction pictures of propane (Ohio Chemical Company-twice redistilled) were taken with the hope of obtaining an accurate carbon-hydrogen distance, since the $\mathrm{C}-\mathrm{H}$ terms in this molecule contribute more than half to the total intensity distribution. The visual appearance of the photographs was that shown in Fig. 2, the relative intensities being an average of estimates by three observers made independently. The photographs were taken at a film distance of $10.43 \mathrm{~cm}$ with electrons of wave-length $0.0605 \mathrm{~A}$. The measured values of ring diameters were obtained from five photographs of varying densities, from which the calculated mean $x_{k}$ 's are tabulated in Table I. A five-term radial distribution calculation $^{11}$ (Fig. 3) gives indication of several important distances in the molecule none

${ }^{11}$ L. Pauling and L. O. Brockway, J. Am. Chem. Soc. 57, 2684 (1935). of which can be taken too seriously, however, because of the overlapping of peaks. In the following calculations all the interaction terms were taken into account except those due to $\mathrm{H}-\mathrm{H}$ when bonded to different carbon atoms. The slight variations in some $\mathrm{C}-\mathrm{H}$ terms due to rotations of the methyl groups about $\mathrm{C}-\mathrm{C}$ bonds were also neglected. That these approximations are legitimate can be seen from Fig. $4 A$ in which the dotted line indicates the theoretical intensity distribution when all factors are considered while the full curve, when the enumerated simplifications are made. The three independent parameters taken were the carbon-hydrogen, carboncarbon when bonded, and carbon-carbon $(e n d)$

TABle I.

\begin{tabular}{cccc}
\hline \hline & $x \kappa$ & $\begin{array}{c}\text { RELATIVE } \\
\text { WTS. }\end{array}$ & $\begin{array}{c}\text { RELATIVE } \\
\text { INTENSITIES }\end{array}$ \\
\hline 1 & $(3.150)$ & 1 & 4.0 \\
2 & $(4.488)$ & 1 & 3.6 \\
2 & 6.161 & 4 & 8.0 \\
3 & 7.732 & 3 & 2.9 \\
3 & 9.354 & 5 & 3.2 \\
4 & 11.29 & 5 & 0.5 \\
4 & 12.96 & 5 & 1.2 \\
5 & 15.57 & 4 & 0.0 \\
5 & 17.62 & 4 & 0.4 \\
\hline
\end{tabular}




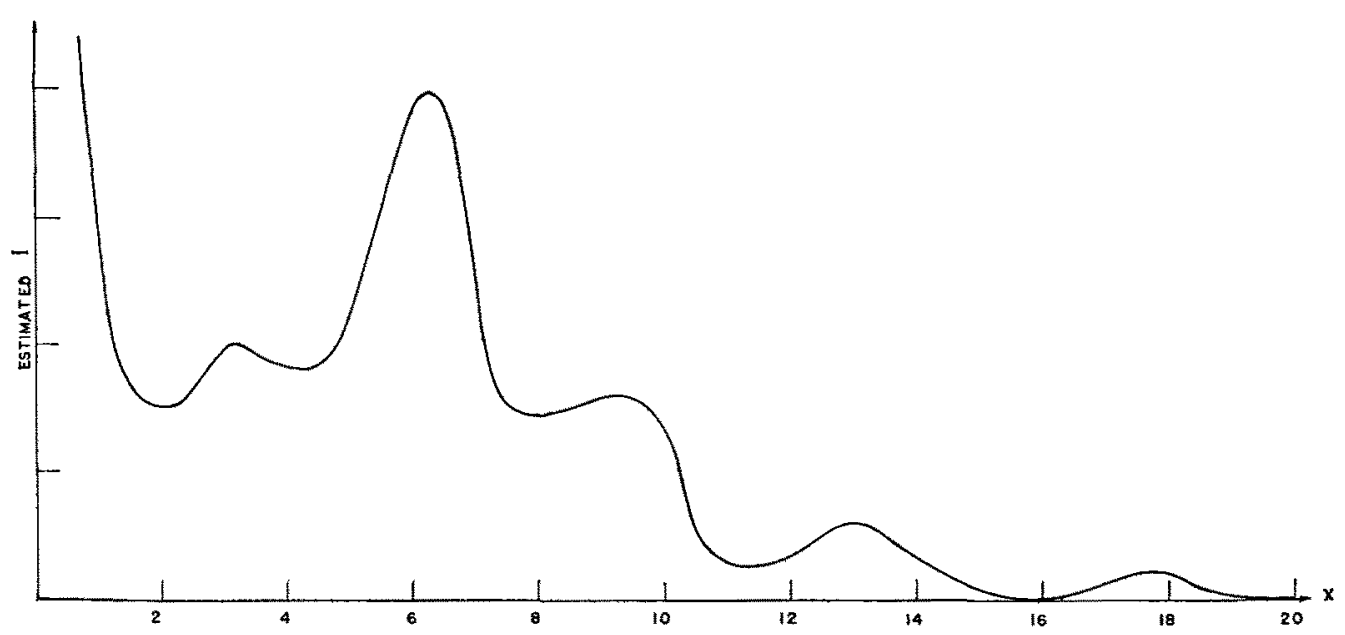

FIG. 2. Visual appearance of electron diffraction photographs of propane.

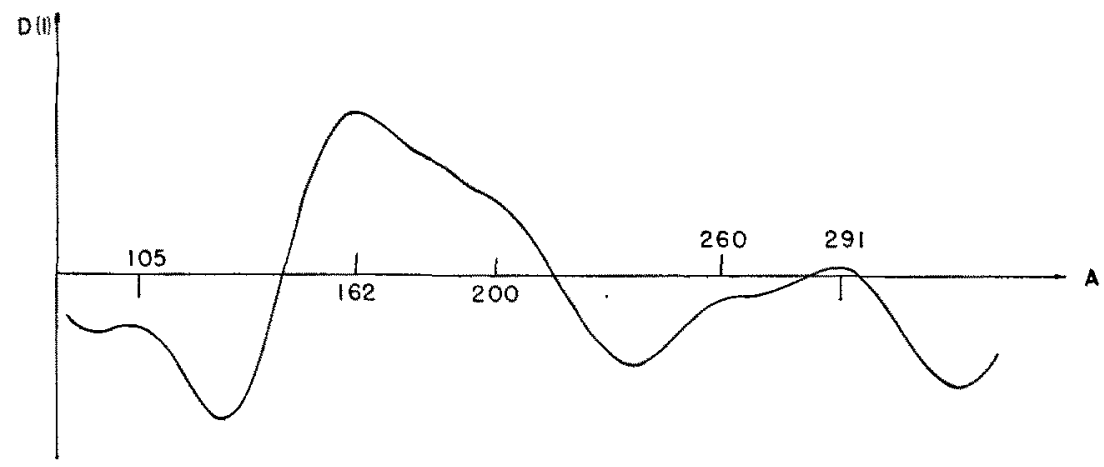

Fro. 3. Radial distribution curve of propane.

distances. The $\mathrm{C}-\mathrm{C}-\mathrm{H}$ angle was assumed tetrahedral throughout. Least-squares treatment of six rings ${ }^{12}$ weighted as shown in Table I gave the following distances:

$$
\begin{array}{cl}
\mathrm{C}-\mathrm{C}_{\text {bonded }} & 1.50_{3} \pm 0.02 \mathrm{~A} \\
\mathrm{C}-\mathrm{C}_{\text {end }} & 2.52_{4} \pm 0.03 \mathrm{~A} \\
\mathrm{C}-\mathrm{H} & 1.08_{1} \pm 0.02 \mathrm{~A} \\
(\mathrm{C}-\mathrm{C}-\mathrm{C}) & 114^{\circ}{ }_{12^{\prime}}
\end{array}
$$

When the last ring is also included in the calculations, an angle of approximately $116^{\circ}$ is indicated. In view of the accepted values, these appear somewhat unusual. ${ }^{13}$ Further support can

12 The inner two were not used because of the St. John effect; the last, because of the inherent uncertainty in the measurement because of its faintness.

${ }^{13}$ For propane, R. Wierl (Ann. d. Physik 13, 453, 1932) gave $\mathrm{C}-\mathrm{C} 1.52 \pm 0.05 \mathrm{~A}$ using the then accepted band spectral value for $\mathrm{C}-\mathrm{H}, 1.10 \mathrm{~A}$. In aliphatic hydrocarbons the accepted $\mathrm{C}-\mathrm{C}$ distance is $1.54 \mathrm{~A}$ and $\mathrm{C}-\mathrm{H}$ distance is be presented in the traditional manner making use of the completed intensity distributions computed by means of equation (2) for a number of models (Fig. 4). Attention is called to curves $A$ and $B$ which indicate the sensitivity of the intensity to changes in the $\mathrm{C}-\mathrm{H}$ distance. Although the differences are not highly pronounced, curve $D$ is evidently in better agreement both in general form and in the positions of the extremal values than any of the others. The lack of a more distinct first maximum may be due to the neglect

$1.06 \mathrm{~A}$ (Table of covalent radii, note 5 ). The present band spectral value for $\mathrm{C}-\mathrm{H}$ from the moment of inertia of methane is $1.11 \mathrm{~A}$ while somewhat different considerations lead to a moment of inertia for methyl deuteride which requires the $\mathrm{C}-\mathrm{H}$ distance to be $1.093 \mathrm{~A}$. See $\mathrm{M}$. Johnson and D. M. Dennison, Phys. Rev. 48, 868 (1935) and N. Ginsburg and E. F. Barker, I. Phvs. Chem. 3, 668 (1935), respectively. The above discrepancy is discussed by the former authors. 


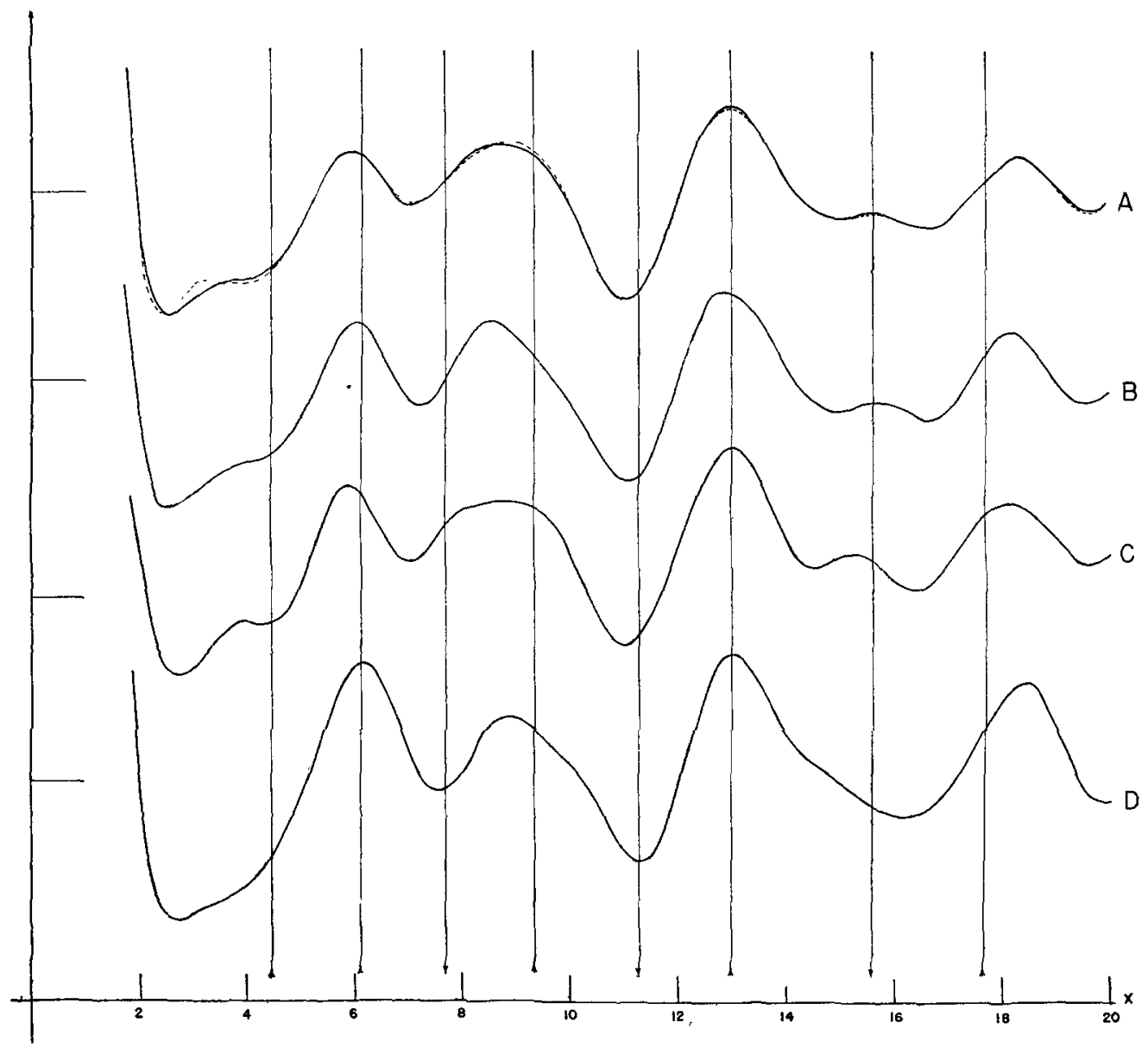

FIG. 4. Calculated intensity curves for models of propane.
A. $\mathrm{C}-\mathrm{H} 1.05$
$\begin{array}{ll}\mathrm{C}-\mathrm{C} & 1.54 \\ \mathrm{C}-\mathrm{C} & 1.54\end{array}$
$\angle \mathrm{C}-\mathrm{C}-\mathrm{C} 109^{\circ} 28^{\prime}$
B. $\mathrm{C}-\mathrm{H} 1.06$
C. $\mathrm{C}-\mathrm{H} 1.06$
$\mathrm{C}-\mathrm{C} 1.54$
$\mathrm{C}-\mathrm{C} 1.50$
$\angle \mathrm{C}-\mathrm{C}-\mathrm{C} 109^{\circ} 28^{\prime}$
$\angle \mathrm{C}-\mathrm{C}-\mathrm{C} 112^{\circ}$
$\angle \mathrm{C}-\mathrm{C}-\mathrm{C} 114^{\circ}$

of the $\mathrm{H}-\mathrm{H}$ terms. One must keep in mind, when looking at these curves, that they are to be superposed on a monotonic decreasing function of $x$ because of the presence of the structure factor terms.

Although the present photographs favor strongly the distances quoted, the writer hesitates to present them as final values. Because of the approximations which must be made in the visual method and the subsequent analysis, the inherently smudged diffraction pattern which propane gives does not warrant the use of further successive approximation calculations.

The author acknowledges his indebtedness to Dr. L. O. Brockway for the use of his apparatus and to Professor L. Pauling and Dr. J. Sherman for helpful discussions pertaining to the material here presented. 